\title{
NDE of Micro Structured Materials By X-Ray Diffraction and Refraction Topography
}

\author{
Manfred P. HENTSCHEL, Axel LANGE, Joerg SCHORS \\ Federal Institute for Materials Research and Testing (BAM), Berlin, Germany
}

\begin{abstract}
For the purpose of micro structural characterization X-ray topography reveals the spatially resolved scattering of materials and small components. It combines the advantages of radiographic imaging and the analytical information of wide and small angle X-ray scattering like phase distribution, texture, micro cracks, interfaces and pores. Scanning techniques at selected scattering conditions permit the topographic characterization of any crystalline or amorphous solid or liquid. Topographic methods and applications for the purposes of research, quality control and damage evaluation are presented.
\end{abstract}

\section{Introduction}

Major tasks within the field of non-destructive characterization of materials are related to the finding and application of structure/property relations like correlations between atomic, nano and micro structures with macroscopic properties. X-ray topography contributes to this, as the structural selectivity covers a range of six orders of magnitude, from $0,1 \mathrm{~mm}$ to $0,1 \mathrm{~nm}$, based on different scattering effects. The spatial resolution up to $10 \mu \mathrm{m}$ is achieved by scanning techniques under pre-selected scattering conditions. The simultaneous detection of single defects covers classical NDT tasks as well. Classical X-ray topography has been applied to single crystals since several decades [1]. Those methods however do not permit the inspection of the more common polycrystalline materials.

The mechanical properties of materials are significantly determined by crystal dimensions, interface topology, micro cracks, pores and texture. X-ray topography can easily contribute to reveal such correlations and exploit them for materials development, for quality assurance or micro damage analysis. In the following the more universal X-ray topographic methods are explained and several applications are reported.

\section{Principles of X-ray Topography}

The extension of single crystal X-ray topography to poly-crystals, amorphous solids and liquids has requested some new methods. X-ray topography is based on Wide Angle X-ray Scattering (WAXS) and Small Angle X-ray Scattering (SAXS) respectively, as well known from classical crystallography. Typical sources are X-ray tubes for fine structure analysis of monochromatic radiation of up to $17 \mathrm{keV}(\mathrm{Mo}-\mathrm{K}-\alpha)$.

Wide Angle X-ray Scattering (WAXS) (diffraction) is sensitive to the atomic and molecular structure far below nanometers dimensions2. The Scherrer diffraction patterns of carbon and polymer fibers are typical examples of textured molecular scattering, which is a 
good precondition for orientation measurements (Fig. 1). The line spectra are analytical probes for the chemical and molecular constituents.

Small Angle X-ray Scattering (SAXS) is a classical tool of colloid, polymer and biological research. Particle dimensions smaller than $50 \mathrm{~nm}$ are determined by 'Guinier' and 'Porod' analysis (diffraction) [3], larger ones by refractometry [4, 5]. Similar to WAXS of textured materials, SAXS of fibers and wires reveals their orientation, as they refract Xrays like cylindrical lenses deflect light. Typical scattering patterns of composite fibers are given by Fig. 1.

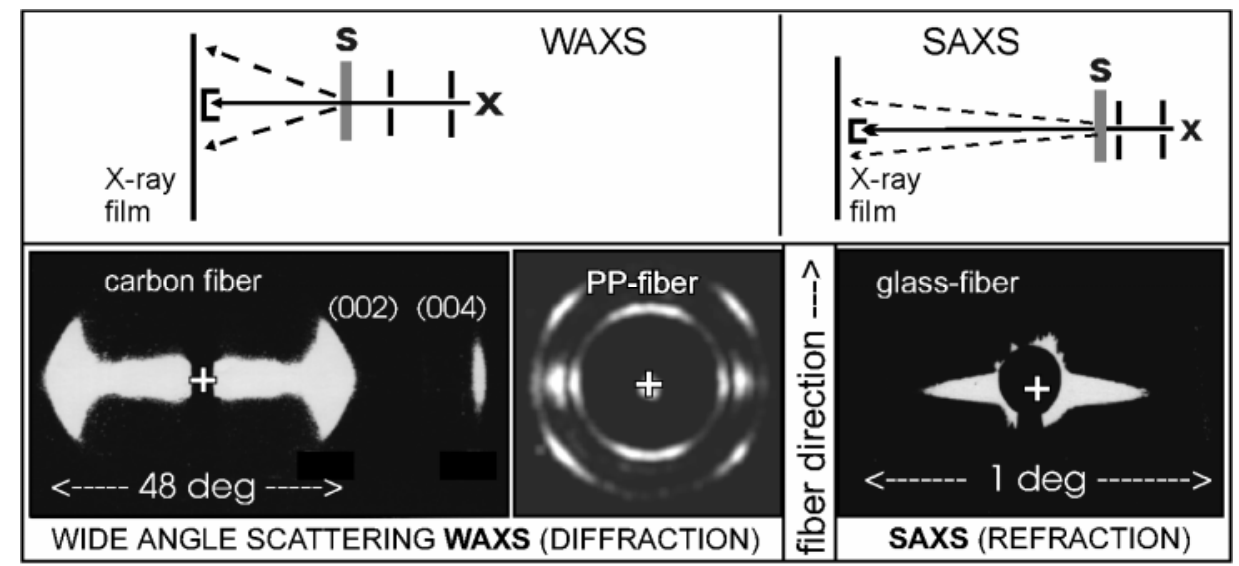

Fig. 1. Beam geometry and oriented wide (WAXS) and small angle (SAXS) X-ray scattering patterns of fibers (vertical).

In order to image the spatial distribution or orientation of the structures, a sample is scanned, while only a selected sector of the scattering pattern is pointing at an X-ray detector window. Fig. 2 shows the basic beam and scan arrangements for different topographic methods of selective structural contrast and projections. They have in common, that the scattering geometry is first pre-selected, then the sample is scanned within two dimensions, rotation included. A standard PC controls the X-ray counters and the micro drives. The high quantum efficiency of scintillation counters permits measurements at reasonable speed, but relatively broad collimation slits (at reduced angular resolution) have to be used in some cases.

\section{X-Ray Topography by wide angle scattering}

\subsection{Two-dimensional X-Ray Scanning Topography}

Scanning topography applies X-ray scattering of the periodic molecular structure into a discrete set of diffraction angles. According to Fig. 2 (top, left) a scattering angle and direction is selected (typically between 5 and 50 deg.) and the sample is scanned within the surface plane. The intensity at each scan position is stored by the computer. The reconstructed computer image reveals the spatial distribution (projection) of the material according to the setting of the scattering angle interval. The primary beam is collimated by a double pinhole of one mm diameter. The detected scattering angles and directions are chosen by slits in front of the detector.

Fig. 3 demonstrates the investigation of a high speed brake disk made of carbon fiber reinforced $\mathrm{SiC}(\mathrm{C} / \mathrm{SiC}-\mathrm{CMC})$. The ceramic composite is manufactured via liquid silicon infiltration of $\mathrm{C} / \mathrm{C}-\mathrm{CMC}$ at $1600^{\circ} \mathrm{C}$. The porous glassy carbon matrix reacts with $\mathrm{Si}$ to give a $\mathrm{SiC}$ matrix which protects the carbon fibers against oxidation at high temperatures. The performance of the material depends on the $\mathrm{C} / \mathrm{SiC}$ mass ratio as revealed by 


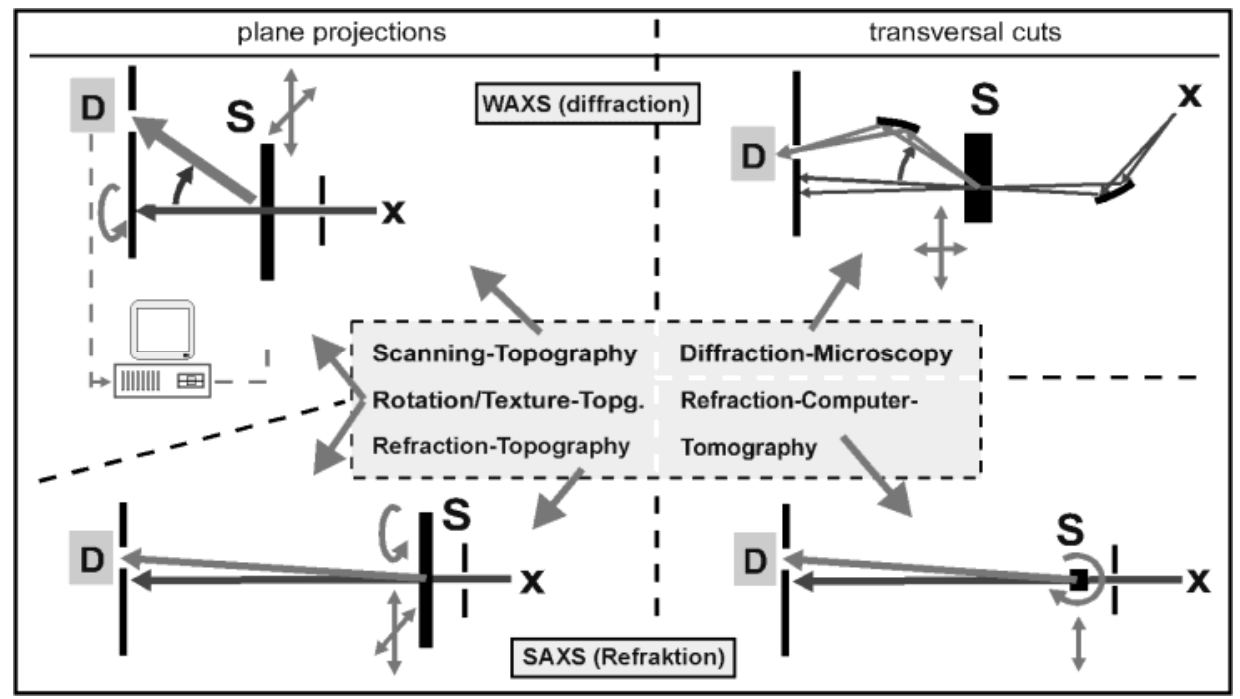

Fig. 2. Beam geometries of X-ray topographic methods by WAXS and SAXS; X: X-ray focus, S: sample, D: detector.

Scanning Topography: The radiographic (inverted) image of Fig.3 shows the X-ray transmission intensity due to density variations of a brake disk section. The fine tetragonal structure of $2 \mathrm{~mm}$ periodicity is related to the carbon fiber bundles of the fabric laminate. But it does not explain, whether porosity or the changing SiC/C-fiber ratio creates the density pattern.

The radiograph contains a bright ring sector flaw of unknown origin. The indicated area is inspected by Scanning Topography. Two Scherrer diagrams of the sample are taken at different scattering angle ranges. The limiting scattering angle is selected by an adjustable diaphragm in front of the film plane. One diagram contains the reflections of both, $\mathrm{SiC}$ and carbon fibers, the other one only the carbon 002 reflection (Fig. 3, top).

For topographic scans the film is replaced by a large area detector. The sample is scanned twice at the two scattering angles by $0.5 \mathrm{~mm}$ steps in both dimensions. Scanning speeds between 1 and 10 positions at $1 \%$ signal standard deviation are typical. The two topographs of $(\mathrm{SiC}+\mathrm{C})$ and $\mathrm{C}$ look very similar (Fig. 3, bottom) and similar to the radiograph as the

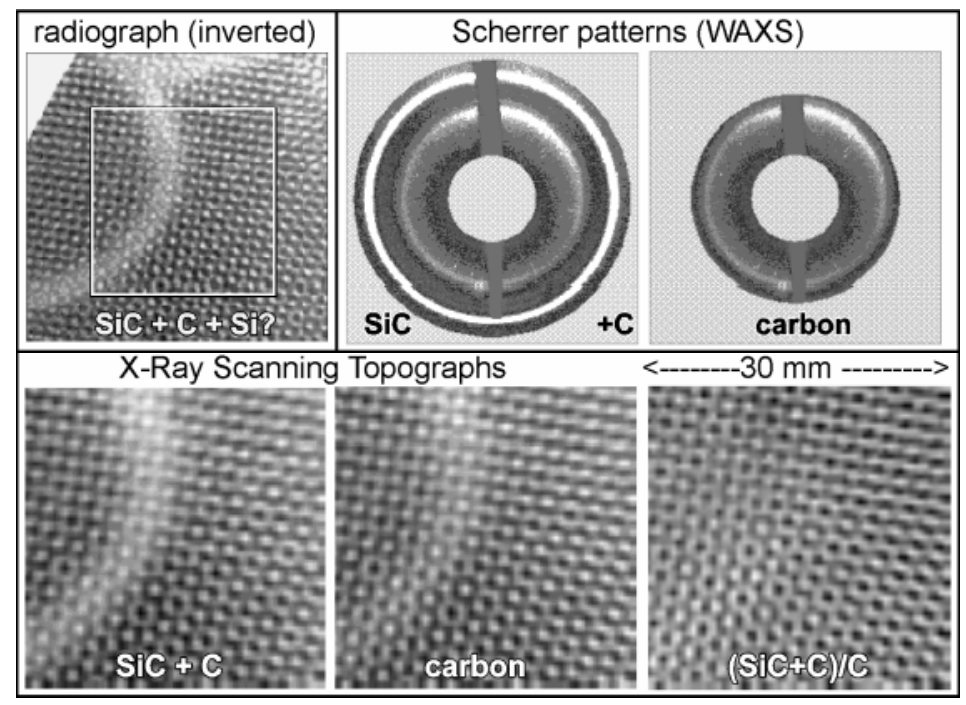

Fig. 3. X-Ray Scanning Topography of C/SiC-CMC; insert of radiograph corresponds to topographs. 
density variation dominates the scattering variation. Solely the ratio of the two topographs is free from density variations, as the absorption effect is identical in both. The resulting ratio image (Fig. 3, bottom right) shows the mass ratio $(\mathrm{SiC}+\mathrm{C}) / \mathrm{C}$. The fine fabric pattern has inverted. Bright positions indicate higher scattering intensity of SiC. They correspond to the dark positions of the topographs (and the radiograph) because of its higher absorption. The ratio image reveals the mixing behavior of the two phases. The absence of the circular ring proves that this is not related to the $\mathrm{SiC}$ or $\mathrm{C}$ content.

\subsection{X-Ray Rotation Topography}

In order to measure the molecular orientation of materials a rotation slit is installed between the sample and a large area detector, as in Fig. 4, left. At each sample position a full rotation of the slit is performed, which reveals the polar intensity distribution by a 'Rotation Profile' within a few seconds. Fig. 4, top right, gives the Rotation Profiles of polypropylene PP and liquid crystal polymer LCP (injection molding samples). The width at half maximum (FWHM) is called the texture angle which is always related to the stiffness and strength of the materials.

Repeated gray scale plots of the Rotation Profiles at different scan positions are added up to Rotation Topographs (Fig. 4, bottom, right). The horizontal coordinate is the rotation angle, the vertical to the sample position. The bright intensity maxima are at the average polymer chain direction (the direction of the highest allowable load on the component). The technique has been applied for quality assessment of helicopter parts [6] and for carbon fiber orientation measurements in composite laminates.

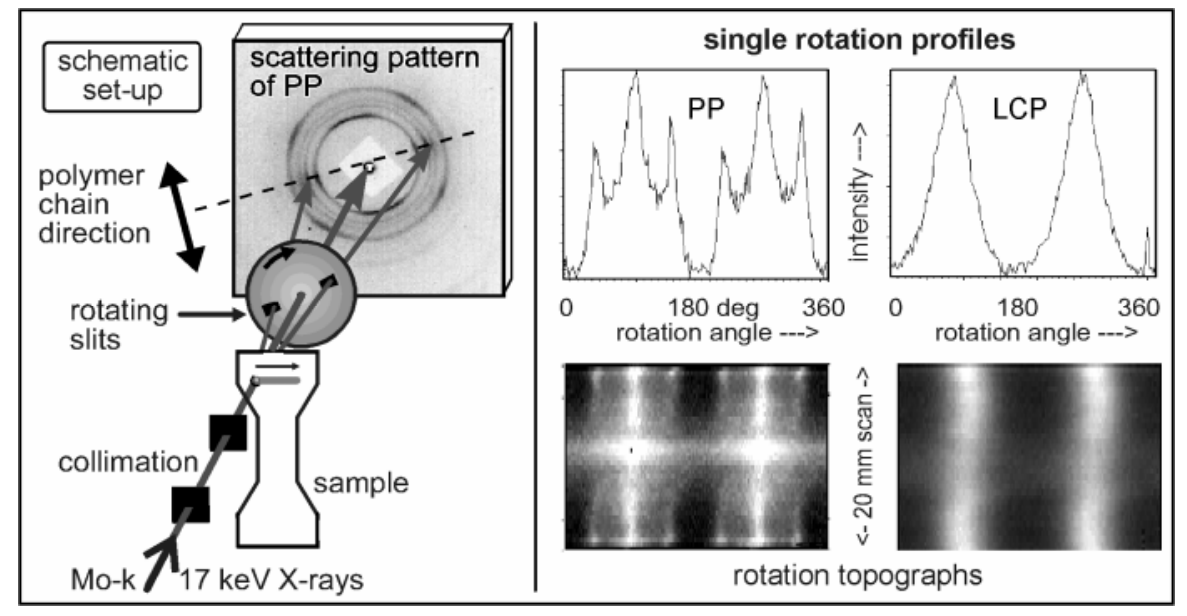

Fig. 4. X-Ray Rotation Topography for polymer chain orientation measurements; Scherrer diffraction pattern of polypropylene PP with rotating slit installed between sample and detection area, left; single rotation profiles and Rotation Topographs, right.

High precision fiber alignment and fiber content is requested for optical mirrors like the 3,5 $\mathrm{m}$ reflector of the projected Far Infrared Space Telescope (FIRST) of ESA (Fig. 5, left). In order to omit the deformation of the optical reflector during mission the allowance for the deviation from the designed fiber direction of the CFRP (carbon fiber reinforced epoxy) sandwich is only $0.1^{\circ}$. Differences between in the fiber mass of each direction are critical as well.

Fig.5 shows at the experimental set-up and the diffraction image of the CFRP sandwich (center), composed of two 6 ply high modulus $C$ fiber composite laminates and a CFRP honeycomb structure inside. The rotation profiles of the fist and second laminate reveal 
each 12 reflection peaks (Fig. 5 right, top). The resulting angular frequency distribution of the 30 degree ply is extracted from 800 rotation profiles on $80 \mathrm{~cm}^{2}$ by computerized curve fit of the rotation profiles (right, bottom). The directions are taken relative to the 0 degree ply. Although the standard deviation exceeds $0.4^{\circ}$, the mean fiber direction deviates only by less than $0.17^{\circ}$. It is determined at 0.05 degree resolution. The relative fiber mass of each direction has been determined from the same rotation profiles (at $1 \%$ resolution per ply). Furthermore it is possible to identify different types of fibers [7].

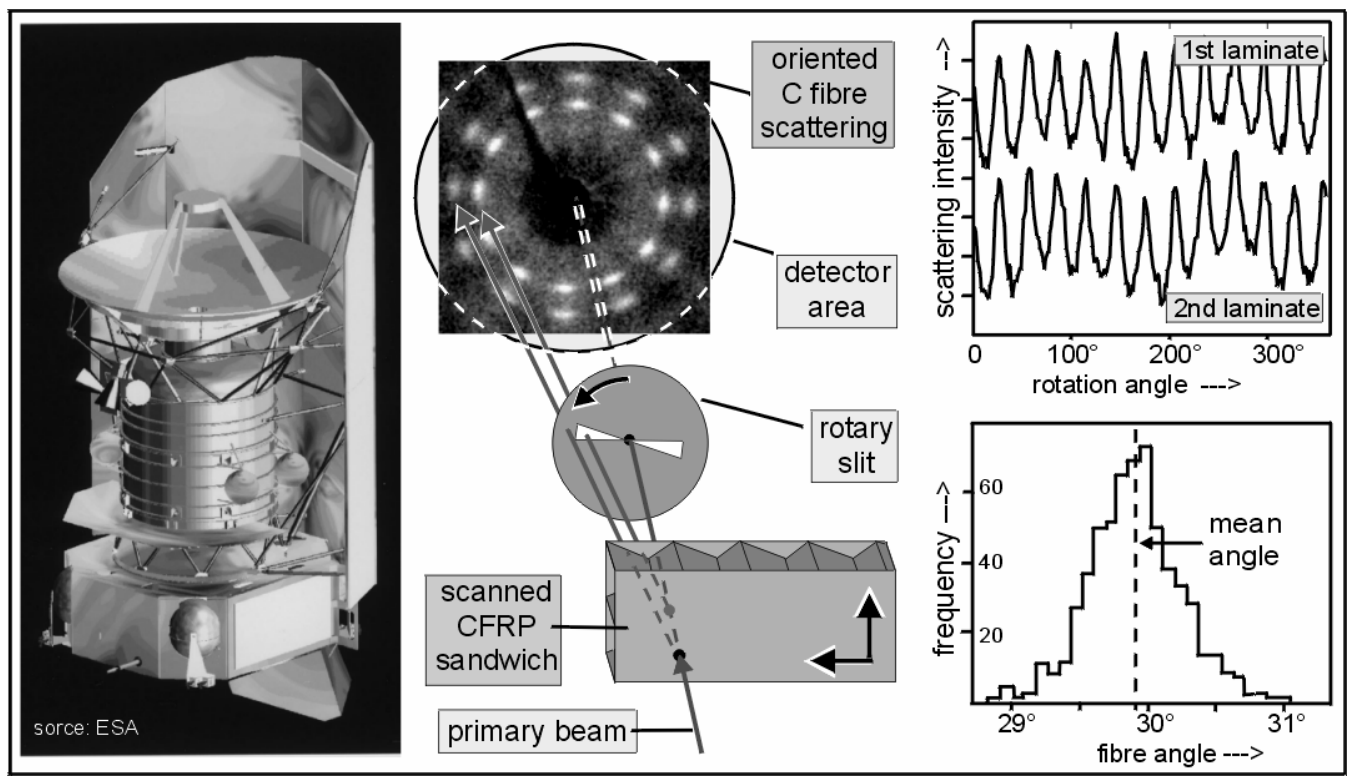

Fig. 5. Rotation topography of CFRP reflector sandwich material for Far Infrared Space Telescope (European Space Agency)

\subsection{X-Ray Texture Topography}

For some practical applications, like evaluating the flow pattern of injection molding parts, it is often sufficient, to image solely the changes in orientation by Texture Topography: The rotation slit is fixed at an inclination angle of maximum slope of the rotation profile. While the sample is scanned any changes in texture and preferred orientation result in intensity changes. The Texture Topograph of two halves of tensile test samples (liquid crystal polymer (LCP), injection molding) reveal spatial variations in molecular orientation (Fig. 6).

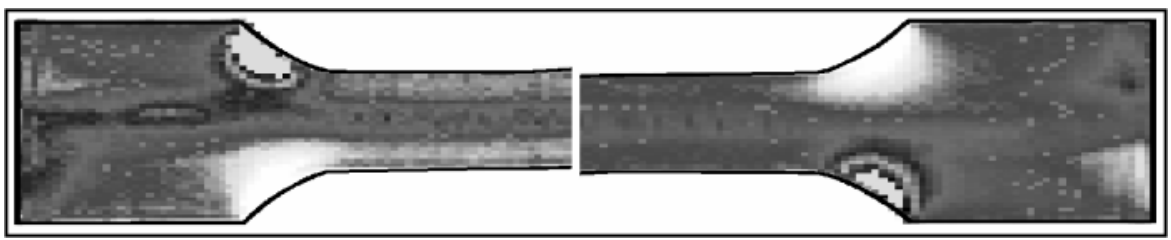

Fig. 6. Texture Topographs of different tensile test samples

(LCP) reveal spatial variations in molecular orientation 


\subsection{X-Ray Diffraction Microscopy}

Topographic imaging can be very significant, especially in the case of thin walled components and when a projection image is sufficient. However an additional transversal resolution would be the ultimate step to gain real three-dimensional information. X-Ray Diffraction Microscopy provides a topographic image of a transversal section. The beam geometry is given in Fig. 2, right, top. The basic principle requests a very narrow beam of the incident and the diffracted rays. For practical use focusing single crystals fulfill this task perfectly, even at an acceptable intensity level. The transversal resolution is $0.4 \mathrm{~mm}$, the lateral resolution is $50 \mu \mathrm{m}$ (Mo-K- $\alpha$ radiation).

By Synchrotron radiation and slit collimation we have reached about $40 \mu \mathrm{m}$ transversal resolution [8]. Fig. 7 shows the transversal cut of a CFRP laminate (vertical fiber direction). The direct localization of the inspection area within en extended component without any rotation (as for computed tomography) is of advantage.

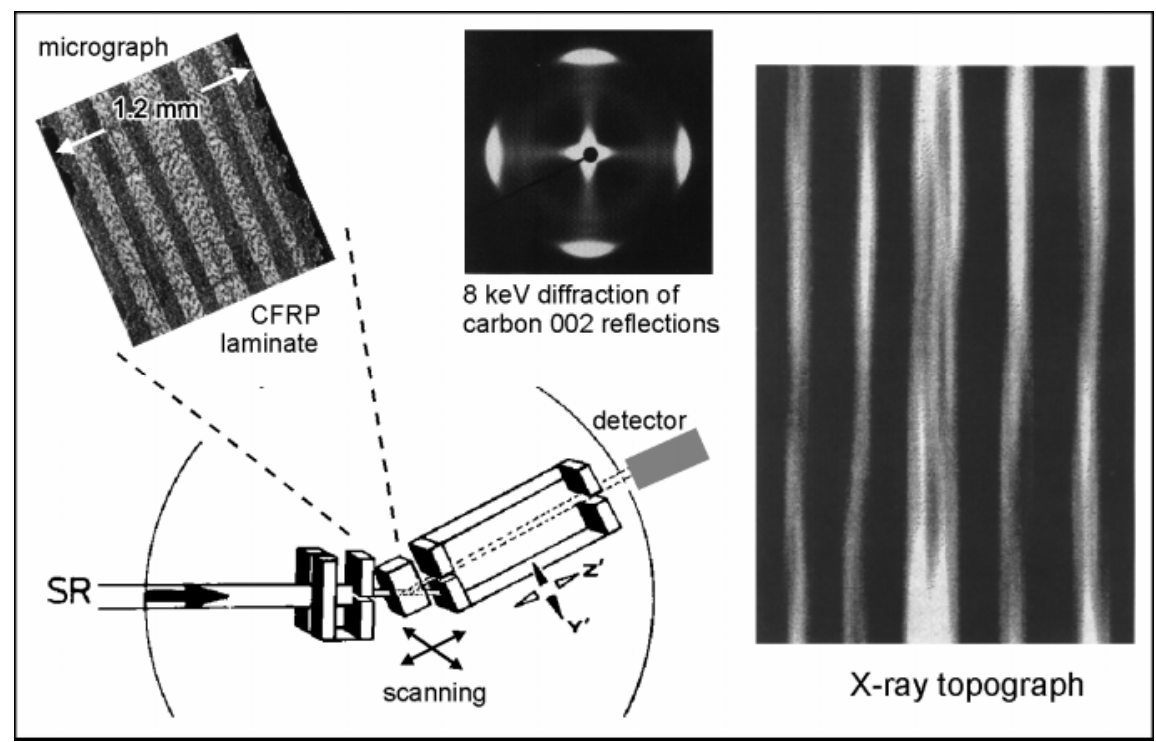

Fig. 7. Synchrotron X-ray Diffraction Microscopy of CFRP laminate; the transversal section (right) images selectively one ply direction as selected by the orientation of the carbon fibre reflections (middle).

\section{X-Ray Topography by Small Angle Scattering}

\subsection{X-Ray Refraction Topography}

Another original development at BAM is X-Ray Refraction Topography. It makes use of the well known optical effect of refraction at interfaces, which for X-rays happens at small scattering angles of a few minutes of arc. In a commercial Kratky camera a very narrow Xray beam crosses a sample, which is scanned in the surface plane and scattering intensities are taken at all positions (Fig. 2, bottom, left). The technique provides nearly linear contrast for specific surfaces and interfaces [9].

In order to gain 2-dimensional images (at a typical spatial resolution of about $20 \mu \mathrm{m}$ $\mathrm{x} 500 \mu \mathrm{m}$ ) a flat sample is scanned across the narrow primary beam. The method is called „X-Ray Refraction Topography“. Usually the strong intensity of the X-ray refraction signal allows scanning the samples within relatively short time. Between 0.1 and 5 seconds per sample position are required for $2 \%$ signal to noise ratio, depending on sample absorption and the inner surface concentration. 
An example of X-Ray Refraction Topography is given by Fig. 8. It shows $\mathrm{SiC}$ ceramic samples, the green body state (left, $44 \%$ porosity) an intermediate state (middle, 16 $\%$ porosity) and the ceramic state (right, $3 \%$ porosity). The porosity is measured precisely by the absorption of the monochromatic primary beam and the refracted intensity is a direct measure of the specific surface. Assuming spherical pore shapes, the ratio of the two independent measurements reveals an average pore size within each scattering volume of 0.1 $\mathrm{mm}^{3}$.

The computer images of Fig.8 represent the spatial mean pore size distributions. Compared to the quite homogeneous green body the pore size is raised in the center area at $1900{ }^{\circ} \mathrm{C}$ while the statistical variance remains within the narrow interval of the green state. After firing at $2180{ }^{\circ} \mathrm{C}$, the mean pore size reaches $2.1 \mu \mathrm{m}$. Now a high variance of $50 \%$ in pore size occurs, although the spatial regularity looks homogeneous. Local clustering occurs due to a considerable disintegration of pores. The decomposition of the statistical arrangement of different pore sizes into clusters of similar pore sizes is a new observation in ceramics.

Other interesting application of Refraction-Topography refer to imaging of the crack density in carbon fiber composites (CFRP) for aerospace applications after fatigue loading and thermal aging or the study of single fiber debonding of fiber composites after impact.

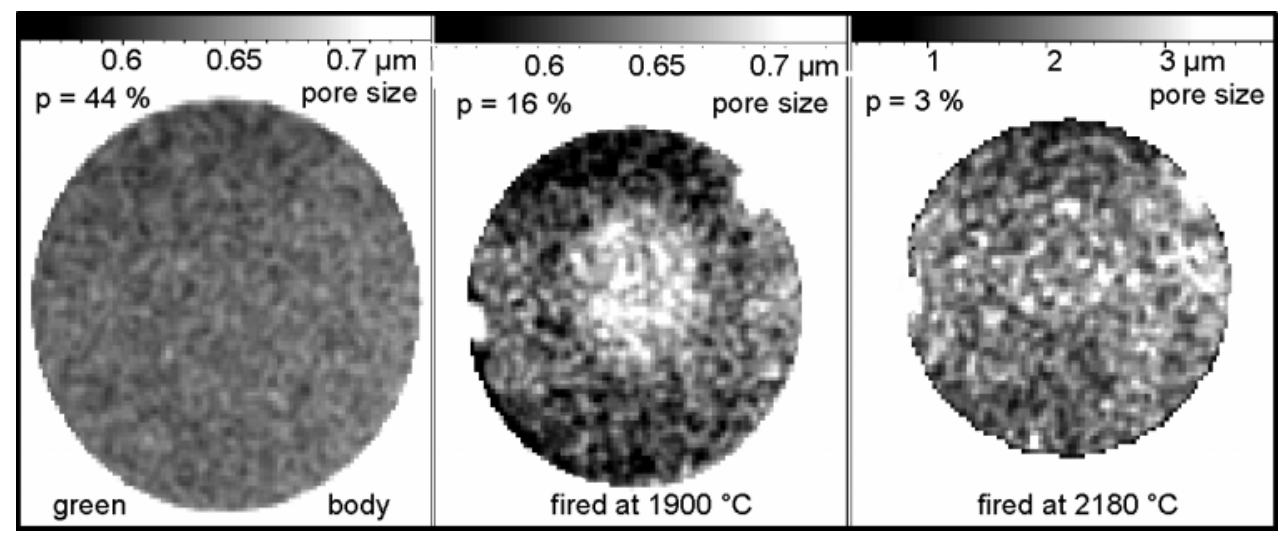

Fig. 8. Refraction Topographs of spatial mean pore size distribution (different gray scales at top!) of SiC before and after sintering at different temperaturesT; p: porosity

\subsection{X-Ray Refraction Computed Tomography}

Refraction-Topography images the projection of the specific surface, but a transversal section image may be desired in order to reveal details. This is achieved by a combination of Refraction line scans and Computed Tomography techniques according to the beam geometry of Fig. 2 (bottom right). A section of a C/C ceramic matrix (CMC) composite is investigated in order to image the different crack patterns developing during pyrolysis at three different levels of fiber debonding.

For a classical (absorption) density map by Computed Tomography (CT) multiple linear scans of the sample are repeated at different rotation angles at zero scattering angle. The absorption signals are reconstructed by „parallel beam filtered back projection“ as shown by Fig. 9, center. The resulting density tomograph reveals only major cracks.

If the procedure is repeated at a scattering angle of two minutes of arc, the interface image (Fig. 9, right) contrasts much finer cracks. The average intensity levels reveal the quantitative crack density [10]. 

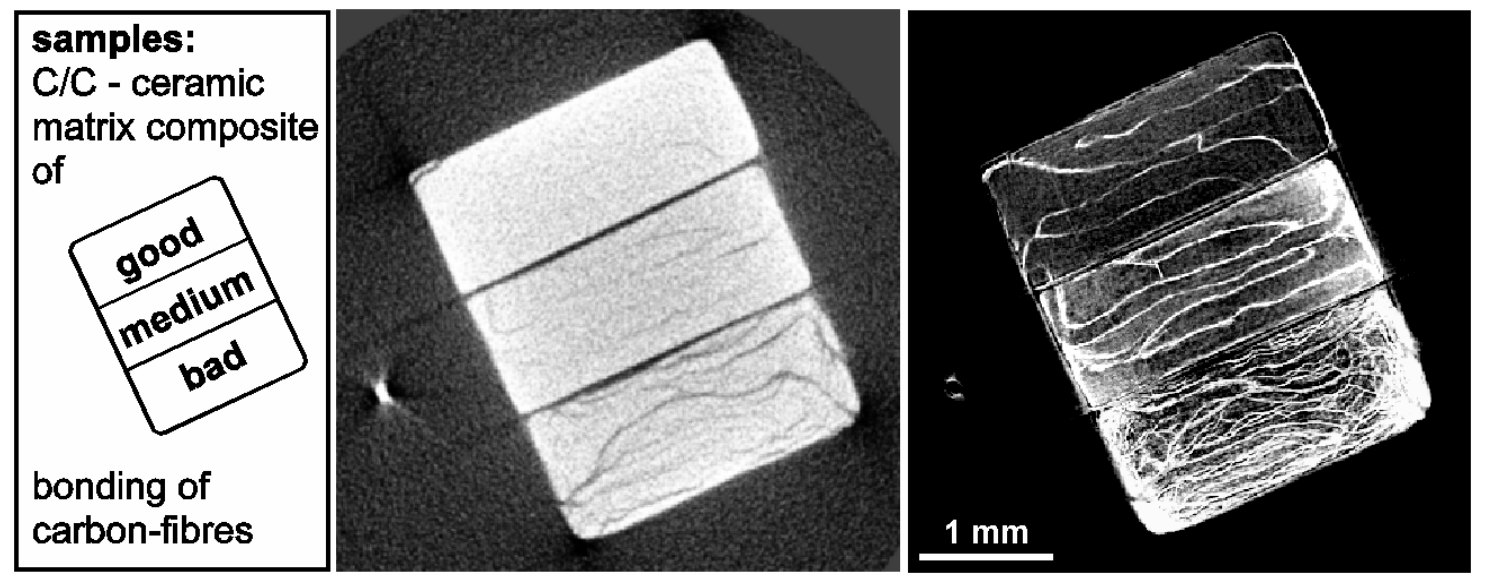

Fig. 9. X-ray Computed Tomography (CT) of Carbon/Carbon ceramic matrix composite (C/C-CMC); left: sample arrangement, middle: conventional (X-ray absorption) CT, right: cracks by Refraction Computed Tomography

\section{Further potential}

The methods of X-Ray Topography request preferably the application of 'soft' X-rays below $20 \mathrm{keV}$. As their absorption in metals is high the restriction to light weight materials is recommend in most cases. The most frequent requests for topographic investigations come from industrial developments of polymer processing, plastic foams, composites, ceramics and special papers. Harder radiation of higher brilliance at synchrotron radiation facilities provides faster topographic measurements and higher resolution.

\section{References}

[1] A.R. Lang, “Topography, X-ray diffraction”, The Encyclopedia of X-rays and Gamma Rays, G. L. Clark (Ed), 1053, Reinhold Publishing Corporation, New York, 1963

[2] M. v. Laue, Röntgenstrahlinterferenzen, Akademische Verlagsgesellschaft, Frankfurt/M., 1960

[3] G. Porod, “Die Röntgenkleinwinkelstreuung von dichtgepackten kolloidalen Systemen, Teil I”, Kolloid.Z. 124, 83-114, 1951

[4] M.P Hentschel, R. Hosemann, A. Lange, B. Uther, R. Brückner, "Röntgenkleinwinkelbrechung an Metalldrähten, Glasfäden und hartelastischem Polypropylen”, Acta Cryst. A 43, 506-513, 1987

[5] M.P. Hentschel, K.-W. Harbich, A. Lange, "Non-destructive evaluation of single fiber debonding by Xray refraction”, NDT \& E international, 27, 275-280, 1994

[6] M.P. Hentschel, A. Lange, K.-W. Harbich, D. Ekenhorst, J. Schors, "Röntgentopographie der Faser- und Polymerorientierung”, Materialprüfung/materials testing 39, 121-123, 1997

[7] M.P. Hentschel, A. Lange, J. Schors, T. de Rooij, M.D. Judd, "Precise fibre direction and content in CFRP measured by X-ray rotation topography” Proc. of the Europ. Conf. on Spacecraft Structures, Materials and Mechanical Testing, Noordwijk, 29. 11.-01. 12. 2000, B. Schürmann (Ed), ESA SP-468, 129134, ESA, Noordwijk, NL, 2001

[8] J.D. Stephenson, M.P. Hentschel, A. Lange, "Synchrotron radiation (hard) X-ray diffraction microscopy of carbon fiber reinforced plastic (CFRP)”, Nucl. Instr. and Methods in Physics Research, B 88, 287-292, 1994

[9] U. Mücke, K.-W. Harbich, T. Rabe, "Determination of pore sizes on sintered ceramic materials using image analysis and X-ray refraction”, Ceramic forum international / Ber. d. DKG, 74, 95-98, 1997

[10]M.P. Hentschel, A. Lange, R.B. Müller, J. Schors, K.-W. Harbich, "RöntgenrefraktionsComputertomographie”, Materialprüfung/materials testing 42, 217-221, 2000 